\title{
CALIDAD DE VIDA EN ADULTOS POSINFARTADOS DE LA UNIDAD CARDIOVASCULAR DEL HOSPITAL DR. HERNÁN HENRÍQUEZ ARAVENA DE TEMUCO
}

\author{
QUALITY OF LIFE IN ADULT POSTINFARCTION PATIENTS OF THE \\ CORONARY UNIT AT DR. HERNÁN HENRÍQUEZ ARAVENA HOSPITAL \\ FROM THE CITY OF TEMUCO
}

\author{
Ana María López Beltrán* \\ SARA BARrios CASAS ${ }^{* *}$ \\ Jorge Cortés QuintANA ${ }^{* * *}$
}

\begin{abstract}
RESUMEN
Objetivo: Determinar la Calidad de Vida Relacionada con Salud (CVRS) de los pacientes infartados adultos de la Unidad Cardiovascular del Hospital Dr. Hernán Henríquez Aravena de la ciudad de Temuco, Chile. Material y método: Estudio cuantitativo exploratorio transversal retrospectivo. 105 usuarios posinfartados participaron en el estudio, correspondiendo a $72,3 \%$ varones y $27,6 \%$ mujeres; el $57,1 \%$ tenía escolaridad básica completa; el 47,6\% un nivel de ingresos inferior a $\$ 200.000$ y una edad media de 57,3 (DT 2,09). El instrumento utilizado fue el Cuestionario de Salud SF-36. Se aplicó Intervalo de confianza de 95\% y pruebas de multicomparación con el método de Bonferroni. Para el análisis de las 8 dimensiones se utilizó la variable edad como covariable para mejorar la predictibilidad de los efectos. Resultados: Se apreció una peor percepción de CVRS en mujeres en los ítems de Función física, Dolor corporal, Salud General, Vitalidad, Función Social y Salud Mental; esto significa una peor evaluación del Dominio físico y mental en estas participantes. Las subescalas mejor evaluadas en varones corresponden a Desempeño emocional, Vitalidad, Función física y Salud mental. Sólo en las escalas Rol Físico Social y Rol Emocional, el factor sexo no generó diferencias estadísticamente significativas. Conclusión: Al determinar la CVRS en los sujetos del estudio, se puede concluir que existe una percepción mejor en los varones que en las mujeres enroladas en este estudio, tanto en el dominio físico como mental.
\end{abstract}

Palabras clave: Calidad de vida, enfermedad coronaria, infarto agudo de miocardio, enfermería cardiovascular.

\begin{abstract}
Objective: To determine the Quality of Life Related to Health (HRQL) of the adult infarcted patients of the Cardiovascular Unit at Dr. Hernán Henríquez Aravena Hospital in the city of Temuco, Chile. Material and method: Quantitative retrospective cross-sectional exploratory study. 105 post-infarction users participated in the study, of wich $72.3 \%$ were male and $27.6 \%$ female; $57.1 \%$ of then had completed elementary education; $47.6 \%$ had an income of less than $\$ 200,000$ and an average age of 57.3 (DS 2.09). The instrument used was the SF-36 Health Questionnaire. 95\% Confidence Interval and multicomparison tests were applied by using the

\footnotetext{
*Enfermera. Magíster en Enfermería mención Gestión del cuidado. Universidad de La Frontera. Académica Escuela Enfermería, Facultad de Ciencias de la Salud, Universidad Central de Chile. Email: ana.lopez@ucentral.cl

${ }^{*}$ Enfermera. Dra. en Enfermería. Académica Depto. de Enfermería, Facultad de Medicina, Universidad de La Frontera. Temuco, Chile. Email: sara.barrios@ufrontera.cl

${ }_{* * *}^{*}$ Profesor Universitario. Doctor @ Estadística. Universidad de Salamanca. Académico Departamento Matemática y Estadística Universidad de La Frontera. Temuco, Chile. Email: jorge.cortes@ufrontera.cl
} 
Bonferroni method. For the analysis of the 8 dimensions the age variable was used as a covariate to improve the predictability of the effects. Results: A worse perception of HRQL was observed in women in the Physical Function, Body Pain, General Health, Vitality, Social Function and Mental Health items; this means a worse evaluation of the physical and mental domain in these participants. The best evaluated subscales in males correspond to Emotional Performance, Vitality, Physical Function and Mental Health. Only on the Social Physical Role and Emotional Role scales, the sex factor did not generate statistically significant differences. Conclusion: When determining the HRQL in the study subjects, it can be concluded that there is a better perception by the men than by the women enrolled in this study, both in the physical and mental domain.

Key words: Quality of life, coronary disease, myocardial infarction, cardiovascular nursing.

Fecha recepción: 07/11/14 Fecha aceptación: 04/07/16

\section{INTRODUCCIÓN}

El conocimiento de la Calidad de Vida (CV) adquiere gran importancia para enfermería, ya que permite profundizar en las necesidades de cuidado de las personas, aportando información a la práctica clínica en el contexto de las enfermedades y de los factores psicosociales que en ellas influyen. Los/as enfermeros/as que tienen pacientes bajo su cuidado podrían incorporar estos aspectos a la valoración y la intervención en Enfermería en su plan de cuidados; al mismo tiempo elaborar estrategias cuya labor puede extenderse a la clínica, la atención de Enfermería en domicilio, la rehabilitación, la orientación en salud y la consejería familiar para la adaptación a la enfermedad, todo lo cual contribuirá no sólo al mejoramiento de la CV del paciente y familia, sino también al de la propia comunidad (1).

En el caso de las personas que han sufrido un evento como la cardiopatía coronaria, la cual constituye la primera causa de muerte en la población adulta chilena (2), resulta particularmente relevante referirse a la $\mathrm{CV}$, debido a las secuelas o complicaciones derivadas de la misma luego de recuperarse del episodio agudo de esta enfermedad, significando un importante problema de salud pública, que debe ser abordado de la mejor manera posible, tanto en recursos como en capacitaciones para el personal de salud que atiende a estos individuos, otorgando las mejores posibilidades de sobrevida y rehabilitación de la enfermedad, considerando que se presenta tanto en la población activa del país, adultos jóvenes y de mediana edad, siendo este último el grupo con mayor incidencia de Infarto Agudo al Miocardio (IAM) (2).

La revisión de la literatura da cuenta de una amplia evidencia de la medición de la Calidad de Vida Relacionada con Salud (CVRS) y de su evaluación utilizando el Cuestionario de salud SF-36 (3-5).

Un estudio realizado en España concluyó que actualmente la valoración de la calidad de vida en los pacientes coronarios es una medida útil que permite valorar las expectativas de los pacientes, sobre todo en relación a una atención más personalizada con el propósito de conseguir el objetivo de "añadir vida a los años en lugar de años a la vida" (1). En México se realizó un estudio de Calidad de vida en pacientes pos IAM y sus diferencias según edad, el cual mostró que al menos un $50 \%$ de los individuos estudiados presentó una afectación moderada a severa de su nivel de bienestar, presentando los menores de 65 años deteriorada la dimensión correspondiente a relaciones sociales y comportamiento emocional (6).

En el mismo contexto, en Brasil un grupo de enfermeras comparó la calidad de vida entre personas infartadas y otro grupo que padeció angina inestable, donde se aplicó el cuestionario SF-36, identificando los domi- 
nios asociados a dolor, función física, condición general de salud, salud mental, vitalidad y función social como los más deteriorados en ambos grupos de pacientes (7).

Como es posible observar, la medición de la CV puede desempeñar un papel importante en la generación de medidas de resultado en estudios de efectividad en patología cardiovascular (1).

En Australia se estudió la CVRS en pacientes con IAM a través del cuestionario de salud SF-36 en 430 sujetos a los cuales telefónicamente se les aplicó este instrumento 1 a 2 semanas posteriores a la hospitalización y a los 6 meses del evento. Los individuos más jóvenes presentaron menor puntaje en el componente salud mental (CSM) y mayor en el componente de salud físico (CSF), versus los sujetos de mayor edad, quienes obtuvieron menor puntuación en el CSF y mayor en el CSM. La CVRS baja afecta el proceso de recuperación, disminuye el cumplimiento de los tratamientos y la capacidad para realizar actividades de la vida diaria, aumenta la tasa de hospitalización y pone al paciente en riesgo de complicaciones y la muerte (8).

Por otra parte, las diferencias relacionadas con el género en la CVRS se han divulgado entre los pacientes coronarios, con mujeres que no afrontan tan bien física y psicosocialmente como los hombres, aunque la literatura es inconsistente y no queda claro por qué estas diferencias existen. Por lo tanto, se necesita investigación adicional para investigar la asociación entre edad, género y CVRS para los pacientes coronarios.

En Suecia un estudio tuvo como objetivo examinar la percepción de la enfermedad y su asociación con la CVRS autoinformada, la fatiga y la angustia emocional en pacientes con infarto de miocardio. Se aplicó la versión corta del cuestionario de salud SF 36 a 204 pacientes durante la primera semana de hospitalización y 4 meses después del IAM. La percepción de la enfermedad del paciente cambió con el tiempo, de una percepción más aguda a una más crónica de la enferme- dad, y las creencias sobre el control personal y del tratamiento del IM disminuyeron. Además, estas creencias negativas se asociaron con peores experiencias de fatiga y disminución de la CVRS (9).

En Lituania se evaluó la CVRS en Cardiopatía Coronaria según edad, género y método de tratamiento. Utilizaron el cuestionario SF 36 donde el componente físico mejoró en hombres a los 6 meses y el componente mental mejoró en mujeres a los 6 meses. Posterior a este tiempo de seguimiento no se vieron grandes diferencias. Los pacientes de mayor edad experimentaron mejoría en el componente físico (10).

En Suiza se llevó a cabo una investigación, utilizando el cuestionario SF 36, en donde se examinó la relación entre la calidad de vida relacionada con la salud y el afecto positivo, controlando factores de riesgo cardiovascular tradicionales, variables clínicas y afecto negativo en pacientes con enfermedad coronaria (CHD). El afecto positivo se asoció de forma independiente con el componente de salud mental y físico, después de controlar para variables de control. Adicionalmente se observó que el género moderó la relación entre afecto positivo y componente de salud físico, pero no componente de salud mental (11).

Según los antecedentes descritos, esta investigación tiene por objetivo determinar la Calidad de Vida Relacionada con Salud (CVRS) de los Pacientes Infartados Adultos de la Unidad Cardiovascular del Hospital Dr. Hernán Henríquez Aravena, para el año 2009, utilizando el perfil de Calidad de Vida SF-36.

\section{MATERIAL Y MÉTODO}

Investigación cuantitativa, descriptiva, transversal y retrospectiva. El universo correspondió a 261 pacientes (hombres y mujeres) posinfartados hospitalizados durante el año 
2009. Se reclutaron todos los sujetos que se encontraban disponibles al momento de realizar la investigación, los que fueron 105 pacientes (76 hombres y 29 mujeres). Se realizó revisión de estadísticas de ingreso y egreso de la Unidad Cardiovascular del Hospital Dr. Hernán Henríquez Aravena de la ciudad de Temuco durante el año mencionado.

Los criterios de inclusión correspondieron a: hombres y mujeres (30 o más años) que se encuentren vivos y ubicables a la fecha de realización del estudio; con diagnóstico de IAM con o sin supradesnivel del segmento ST del Electrocardiograma. Los criterios de exclusión correspondieron a: sujetos que hubieran fallecido, pacientes no ubicables, personas analfabetas o intelectualmente incapacitadas e individuos hospitalizados en Unidades de Cuidados Intensivos al momento de aplicar el cuestionario.

La recolección de datos se realizó sobre la base de las fichas clínicas, con las cuales se efectuó un catastro de los pacientes, para ser contactados vía telefónica y ser citados presencialmente en el policlínico de cardiología del hospital, donde la investigadora principal aplicó el instrumento SF-36, versión 1.4 ( $\alpha$ de Cronbach $\geq 0,9$ para los componentes de salud física y mental). Se les explicó detalladamente el instrumento según el total de ítems, las cuales corresponden a 36 preguntas: 10 de Función física, 4 de Rol Físico sobre actividad, 2 de Dolor Corporal, 5 de Salud general, 4 de Vitalidad, 2 de Función Social, 3 de Rol emocional, 5 de Salud mental y una última interrogante diferencial de salud (Salud general hace un año). Además, en la misma entrevista se pesquisaron los datos sociobiodemográficos de los participantes.

El estudio consideró los siete principios éticos de Ezequiel Emanuel (12) para contribuir hacia el bienestar de las personas, incrementando el cuerpo de conocimientos propios de enfermería, garantizando la protección de la identidad y privacidad de los sujetos, además de seleccionarlos equitativamente; las personas enroladas en esta investi- gación firmaron consentimiento informado y tuvieron libre decisión de retirarse cuando quisieran. Asimismo, se contó con la autorización de la Subdirección médica del establecimiento. Además fue presentado a Comité de ética del Hospital Hernán Henríquez Aravena donde fue aprobado para su ejecución.

El análisis de los datos da cuenta de los ítems por dimensión, su significado, número de categorías y su correspondiente especificación, como también la indicación que los ítems deben ser invertidos antes de crear las 8 escalas de primer orden, producto del análisis factorial que aplicaron los autores y creadores de la metodología de análisis (4). Para determinar la CVRS de los pacientes posinfartados, se aplicó Intervalo de Confianza de 95\% y pruebas de multicomparación de variables con el método de Bonferroni. Para el análisis de las 8 dimensiones del instrumento se utilizó la variable Edad como covariable para mejorar la predictibilidad de los efectos.

\section{RESULTADOS}

Destaca que dentro del perfil sociodemográfico del total de participantes, más de un $70 \%$ eran de sexo masculino. El nivel de escolaridad mayoritariamente alcanzado fue de estudios básicos, el nivel de ingresos económicos correspondieron a dos tramos predominantes que comprenden sumas menores a 500 mil pesos mensuales. La edad media es de 57,3 +/- 2,093 años (Intervalo de confianza de 95\%) (Tabla 1).

$\mathrm{Al}$ analizar las subescalas del instrumento se aprecia que la subescala mejor evaluada es RE (Rol Emocional), posteriormente le siguen FS (Función Social), VT (Vitalidad), FF (Función Física) y SM (Salud Mental). Las subescalas de DC (Dolor Corporal) y SG (Salud General) se observan con regular evaluación. Lo peor evaluado es RFS (Rol Físico) (Gráfico 1).

Cuando se comparan las 8 subescalas de 
Tabla 1. Perfil sociodemográfico de los adultos posinfartados egresados el año 2009 de la Unidad Cardiovascular del HHHA $(\mathrm{n}=105)$.

\begin{tabular}{lc}
\hline Variables & Porcentaje \\
\hline Sexo & \\
Hombre & 72,38 \\
Mujer & 27,62 \\
Nivel de estudios & \\
Básica Completa & 57,14 \\
Media Completa & 28,57 \\
Superior Completa & 14,29 \\
Nivel Socioeconómico & \\
$<200$ mil pesos chilenos & 47,62 \\
201 - 500 mil pesos chilenos & 40,95 \\
$>500$ mil pesos chilenos & 11,43 \\
& [IC 95\% 55,278; 59,464] \\
Edad (Media DT) & \\
${ }^{\star}$ Salário Mínimo \$165.000 en Chile al momento del estudio.
\end{tabular}

Gráfico 1. Calidad de Vida en los pacientes adultos postinfartados año 2009 del HHHA según subescalas: Función Física (JFF), Rol Físico (JRFS), Dolor Corporal (JDC), Salud General (JSG), Vitalidad (JVT), Función Social (JFS), Rol Emocional (JRE) y Salud Mental (JSM).

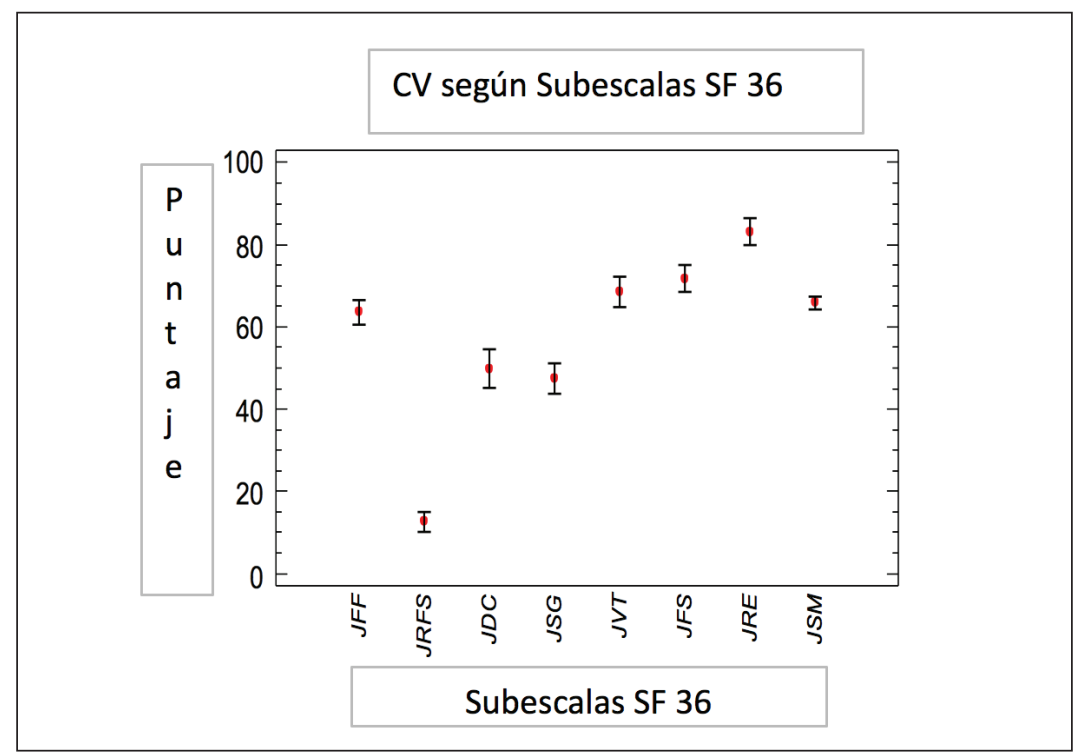

Interpretación puntaje: 0-50 peor percepción de CV; 50 a 100 mejor percepción de CV. 
este instrumento, según sexo, las mujeres evalúan de peor manera la mayor parte de las subescalas que se detallan a continuación, ge- nerando diferencias que son observables en la Tabla 2.

Tabla 2. Diferencia de percepción (puntaje) entre hombres y mujeres con respecto a subescalas de instrumento SF 36.

\begin{tabular}{lccccc}
\hline & \multicolumn{5}{c}{ Sexo } \\
\cline { 2 - 5 } Subescalas de instrumento SF 36 & \multicolumn{2}{c}{ Hombre } & \multicolumn{2}{c}{ Mujer } & Valor p \\
\cline { 2 - 5 } & Media & DT & Media & DT \\
\hline Componente Salud Física & 40 & 23,4 & 30 & 23,7 & 0,000 \\
Función física & 70 & 23,7 & 50 & 25,5 & 0,000 \\
Rol Físico sobre actividad & 60 & 25,5 & 30 & 23,4 & 0,200 \\
Dolor Corporal & 50 & 23,6 & 30 & 20,5 & 0,000 \\
Salud General & 50 & 20,9 & 30 & 20 & 0,000 \\
Componente Salud Mental & 55 & 20,5 & 50 & 22,9 & 0,000 \\
Vitalidad & 70 & 20 & 50 & 21,4 & 0,000 \\
Función social & 70 & 22,9 & 60 & 17,7 & 0,000 \\
Rol Emocional & 60 & 21,4 & 30 & 23,6 & 0,220 \\
Salud Mental & 70 & 17,7 & 60 & 20,9 & 0,000 \\
\hline
\end{tabular}

En el Componente de Salud Física, en las subescalas Función Física (esfuerzos intensos, esfuerzos moderados, limitación para levantar o llevar bolsas de compras, subir uno o varios pisos por escalera, agacharse, arrodillarse, caminar $1 \mathrm{~km}$, etc.), Dolor corporal (incidencia corporal del dolor e incidencia del dolor en las actividades laborales y/o cotidianas) y Salud general las mujeres tienen una peor percepción respecto de los hombres, siendo esta diferencia significativa.

En el Componente de Salud Mental, también las mujeres tienen una peor percepción con respecto a los hombres en las subescalas Vitalidad, Función Social (frecuencia de problemas físicos o emocionales que afectan actividades sociales, la incidencia de problemas de salud física o emocional en actividades sociales) y Salud Mental (nerviosismo, desánimo, tranquilidad, tristeza, felicidad), siendo esta diferencia significativa.
Sólo en las escalas Rol Físico Social y Rol Emocional, el factor sexo no genera diferencias estadísticamente significativas.

Finalmente, los Componentes Físico y Mental fueron mejores evaluados por los hombres, en comparación con las mujeres $(\mathrm{p}=0,000 ; \mathrm{p}=0,000$ respectivamente).

\section{DISCUSIÓN Y CONCLUSIÓN}

Al describir la calidad de vida por medio de las 8 subescalas, se aprecia que la mejor evaluada es RE (Rol Emocional), le siguen FS (Función Social), VT (Vitalidad), FF (Función Física) y SM (Salud Mental). Las subescalas de DC (Dolor Corporal) y SG (Salud General) se observan con regular evaluación. Lo peor evaluado es RFS (Rol Físico). Estos resultados coinciden con los dominios peor 
evaluados (DC, SG) en un estudio en Brasil llevado a cabo por un grupo de enfermeras aplicando el mismo instrumento (7).

Respecto de la utilización del cuestionario SF 36 en Chile, la revisión de la literatura evidencia que la validación de este instrumento fue realizada por la Superintendencia de Salud en dos estudios, donde el N fluctuó entre 1.200 y 4.000 sujetos (5). Sin embargo, otros estudios han sido realizados con muestras muy pequeñas (desde 59 a 159 sujetos de investigación) y en individuos con patologías distintas a la abordada en el presente estudio, por lo que no es posible comparar estos hallazgos con las investigaciones antes mencionadas.

El estudio de Gallani MC et al., donde se aplicó el cuestionario SF 36 a 132 sujetos, concluyó, en concordancia con lo encontrado en el presente estudio, que las mujeres tienen una peor CV en el Componente Salud Mental. También los sujetos de sexo femenino presentaron menor $\mathrm{CV}$ en el Componente físico, especialmente en los ítemes de Función física y Rol físico asociado a la actividad (7).

Los resultados de este estudio coinciden, en parte, con estudios que concluyen que las mujeres tienen peor percepción de CV en el aspecto mental, versus los hombres que presentan mayor deterioro en el componente físico (11, 13-20). Sin embargo, en este estudio las mujeres tienen peor percepción de CV en los aspectos del Componente de Salud física (CSF) y Componente de Salud mental (CSM).

Dentro de las limitaciones de este estudio, se aprecia que al comparar los resultados de los estudios descritos y el realizado en esta investigación, se evidencia que existen diferencias con respecto a los resultados, esto debido al tamaño muestral, a los momentos de aplicación (en este caso sólo en una oportunidad) y a las variables analizadas, ya que los otros estudios incluyen comorbilidad, o incluso aplican distintos instrumentos para realizar un análisis más enriquecedor.
Otra limitación de esta investigación corresponde a la versión utilizada del instrumento, ya que no es la más reciente aceptada en español; se utilizó de la versión 1.4, la cual no corresponde al ejemplar más actualizado, la que corresponde a la versión 2.0, debido a que el acceso y permisos que se obtuvieron fueron de la versión anterior.

Como conclusiones se logró caracterizar a este grupo de estudio y relacionar las variables sociodemográficas antes descritas con la CV de los pacientes incluidos en esta investigación. Además se identificaron algunas falencias existentes en el instrumento SF-36, el cual ha sido ampliamente utilizado en el ámbito sanitario, lo cual da origen a nuevas investigaciones.

Por último, destacar la importancia de integrar a la atención de salud de estos pacientes los componentes físicos, mentales y sociales que se requieren para considerar a los pacientes en todas sus dimensiones como personas, destacando el componente social debido a la reinserción tanto laboral como emocional y afectiva a las que se ven enfrentados estos sujetos, siempre buscando la mantención y mejoría del estado de salud de los individuos que están bajo el cuidado de los/as enfermeros/as y del equipo de salud, logrando así el trabajo interdisciplinario y efectivamente el equipo que se requiere para el bienestar de las comunidades y poblaciones alrededor del mundo.

\section{REFERENCIAS}

1. Torres A, Sanhueza O. Modelo estructural de enfermería de calidad de vida e incertidumbre frente a la enfermedad. Cienc. enferm. 2006; XII(1): 9-17.

2. Slevin ML, Plant H, Lynch D, Drinkwater J, Gregory WM. Who should measure quality of life, the doctor or the patient? Br J Cancer. 1988; 57(1): 109-12.

3. Leplège $A$, Hunt $S$. The problem of qua- 
lity of life in medicine. JAMA. 1997; 278(1): 47-50.

4. Vilagut G, Ferrer M, Rajmil L, Rebollo P, Permanyer-Miralda G, Quintana JM. et al. El Cuestionario de Salud SF-36 español: una década de experiencia y nuevos desarrollos. Gac Sanit [Internet]. Abr 2005 [citado 28 ene 2012]; 19(2): 135-150. Disponible en: http://www.gacetasanitaria. org/es/linkresolver/el-cuestionario-salud-sf-36-espanol/S0213911105713418/

5. Olivares P. Estado de salud de beneficiarios del sistema de salud de Chile: 2004 -2005 [Internet]. Santiago, Chile: Departamento de Estudios y Desarrollo, Superintendencia de Salud; Ene 2006 [citado 28 ene 2012]. Disponible en: http://www. supersalud.gob.cl/documentacion/569/ articles-1062_recurso_1.pdf.

6. Abreu A, Arenas J, Rojas M, Rodríguez M, Rodríguez J. Calidad de vida en pacientes post infarto de miocardio. Diferencias según la edad. Madrid: Pearson; 2003. 29 p.

7. Gallani MC, Colombo RC, Alexandre NM, Bressan-Biajone AM. Quality of life in coronary patients. Rev Bras Enferm. 2003; 56(1): 40-3.

8. Hawkes A, Patrao TA, Ware R, Atherton JJ, Taylor CB, Oldenburg BF. Predictors of physical and mental health-related quality of life outcomes among myocardial infarction patients. BMC Cardiovasc. Disorders [Internet] Sept 2013 [citado 28 mar 2014]; 13: 69. Disponible en: http://www.biomedcentral.com/14712261/13/69.

9. Alsén P, Brink E, Persson LO, Brändström Y, Karlson BW. Illness perceptions after myocardial infarction: relations to fatigue, emotional distress, and health-related quality of life. J Cardiovasc Nurs [Internet]. Mar-Apr 2010 [citado 28 mar 2014]; 2: E1-10. Disponible en: http://www.ncbi.nlm.nih.gov/pub$\operatorname{med} / 20168186$.

10. Staniūtè M, Brožaitienè. Changes in health-related quality of life among patients with coronary artery disease: a 2-year follow-up. Medicina (Kaunas). 2010; 46(12): 843-50.

11. Stauber S, Schmid JP, Saner H, Znoj H, Saner G, Grolimund J, Von Känel R. Health-Related Quality of Life is Associated with Positive Affect in Patients with Coronary Heart Disease Entering Cardiac Rehabilitation. J Clin Psychol Med Settings [Internet] Mar 2013 [citado 28 mar 2014]; 20(1): 79-87. Disponible en: http://www.unboundmedicine.com/medline/citation/22581108/ Health_related_quality_of_life_is_associated_with_positive_affect_in_patients_with_coronary_heart_disease_entering_cardiac_rehabilitation_.

12. Rodríguez E. Comités de evaluación científica para la investigación en seres humanos y las Pautas CIOMS 2002. Acta bioeth. 2004; 10(1): 37-48.

13. Romero E. Confiabilidad del cuestionario de salud SF-36 en pacientes post-infarto agudo del miocardio procedentes de Cartagena de Indias, Colombia. Rev Colom Cardiol. 2010; 17(2): 41-46

14. White ML, Groh CJ. Depression and quality of life in women after a myocardial infarction. J Cardiovasc Nurs. 2007; 22(2): 138-44.

15. Norris CM, Hegadoren K, Pilote L. Depression symptoms have a greater impact on the 1-year health-related quality of life outcomes of women post-myocardial infarction compared to men. Rev Eur J Cardiovasc Nurs. 2007; 6(2): 92-8.

16. Kristofferzon ML, Löfmark R, Carlsson MJ. Coping, social support and quality of life over time after myocardial infarction. J Adv Nurs. 2005; 52(2): 113-24.

17. Baas LS. Self-care resources and activity as predictors of quality of life in persons after myocardial infarction. Dimens Crit Care Nurs. 2004; 23(3): 131-8.

18. Kim MJ, Jeon DS, Gwon HC, Kim SJ, Chang K, Kim HS et al. Health-related quality-of-life after percutaneous co- 
ronary intervention in patients with UA/ NSTEMI and STEMI: the Korean multicenter registry. J Korean Med Sci. 2013; 28(6): 848-854.

19. Rodríguez M, Merino M, Castro M. Valoración psicométrica de los componentes físicos (CSF) y mentales (CSM) del SF-
36 en pacientes insuficientes renales crónicos en tratamiento con hemodiálisis. Cienc. enferm. 2009; XV(1): 75-88.

20. Mierzynska A, Kowalska M, Stepnowska M, Piotrowicz R. Psychological support for patients following myocardial infarction. Cardiol J. 2010; 17(3): 319-324. 